\title{
WAVELETS OF MULTIPLICITY $r$
}

\author{
T. N. T. GOODMAN AND S. L. LEE
}

\begin{abstract}
A multiresolution approximation $\left(V_{m}\right)_{m \in Z}$ of $L^{2}(\mathbf{R})$ is of multiplicity $r>0$ if there are $r$ functions $\phi_{1}, \ldots, \phi_{r}$ whose translates form a Riesz basis for $V_{0}$. In the general theory we derive necessary and sufficient conditions for the translates of $\phi_{1}, \ldots, \phi_{r}, \psi_{1}, \ldots, \psi_{r}$ to form a Riesz basis for $V_{1}$. The resulting reconstruction and decomposition sequences lead to the construction of dual bases for $V_{0}$ and its orthogonal complement $W_{0}$ in $V_{1}$. The general theory is applied in the construction of spline wavelets with multiple knots. Algorithms for the construction of these wavelets for some special cases are given.
\end{abstract}

\section{INTRODUCTION}

Let $r$ be a positive integer and

$$
l^{2}(\mathbf{Z})^{r}:=\left\{\left(s_{1}, \ldots, s_{r}\right): s_{j} \in l^{2}(\mathbf{Z}), j=1, \ldots, r\right\} .
$$

A multiresolution approximation of multiplicity $r$ is a sequence of closed subspaces $\left(V_{m}\right)_{m \in \mathbf{Z}}$ of $L^{2}(\mathbf{R})$ satisfying the following properties:

$$
\begin{gathered}
V_{m} \subset V_{m+1}, \quad m \in \mathbf{Z}, \\
\bigcup_{m \in \mathbf{Z}} V_{m} \text { is dense in } L^{2}(\mathbf{R}) \text { and } \bigcap_{m \in \mathbf{Z}} V_{m}=\{0\}, \\
f \in V_{m} \Leftrightarrow D_{2} f \in V_{m+1}, \quad m \in \mathbf{Z},
\end{gathered}
$$

where $D_{a} f(x):=f(a x), x \in \mathbf{R}$, for any positive number $a$,

$$
f \in V_{m} \Leftrightarrow T_{2^{-m_{n}}} f \in V_{m}, \quad(m, n) \in \mathbf{Z}^{2},
$$

where $T_{\tau} f(x):=f(x-\tau), x \in \mathbf{R}$, for any $\tau \in \mathbf{R}$,

$$
\text { there exists an isomorphism } \mathscr{I}: V_{0} \rightarrow l^{2}(\mathbf{Z})^{r}
$$

which commutes with the action of $\mathbf{Z}$.

Condition (1.5) means that for any $k \in \mathbf{Z}, \mathscr{I} T_{k}=t_{k} \mathscr{I}$, where $t_{k}: l^{2}(\mathbf{Z})^{r} \rightarrow$ $l^{2}(\mathbf{Z})^{r}$ is a translation operator defined

$$
t_{k}\left(\left(s_{1}(n), \ldots, s_{r}(n)\right)\right)=\left(\left(s_{1}(n-k), \ldots, s_{r}(n-k)\right)\right), \quad\left(s_{1}, \ldots, s_{r}\right) \in l^{2}(\mathbf{Z})^{r} .
$$

Received by the editors January 31, 1992.

1991 Mathematics Subject Classification. Primary 41A15, 41A30, 42C05, 42C15.

Key words and phrases. Wavelets, multiplicity, decomposition and reconstruction algorithms, duality principle, Riesz basis, cardinal splines with multiple knots. 
If $r=1$, the multiresolution approximation will be called simple.

For a given simple multiresolution approximation $\left(V_{m}\right)_{m \in \mathbf{Z}}$, Mallat [9] has given a general construction of wavelets $\phi \in V_{0}$ and $\psi \in W_{0}$, the orthogonal complement of $V_{0}$ in $V_{1}$, such that $\left(T_{n} \phi\right)_{n \in \mathbf{Z}}$ and $\left(T_{n} \psi\right)_{n \in \mathbf{Z}}$ are orthonormal bases of $V_{0}$ and $W_{0}$ respectively. Furthermore, if

$$
\phi_{m, n}(x):=\sqrt{2^{m}} \phi\left(2^{m} x-n\right), \quad(m, n) \in \mathbf{Z}^{2},
$$

then for each $m \in \mathbf{Z},\left(\phi_{m, n}\right)_{n \in \mathbf{Z}}$ is an orthonormal basis of $V_{m}$, and if

$$
\psi_{m, n}(x):=\sqrt{2^{m}} \psi\left(2^{m} x-n\right), \quad(m, n) \in \mathbf{Z}^{2},
$$

then $\left(\psi_{m, n}\right)_{n \in \mathbf{Z}}$ is a complete orthonormal set in $W_{m}$, the orthogonal complement of $V_{m}$ in $V_{m+1}$.

In [6] we have extended Mallat's results to multiresolution approximation of multiplicity $r$, for any positive integer $r$, where a relationship between wavelets and the concept of wandering subspaces in operator theory (see Halmos [7, Problem 155], and Robertson [10]) was exploited to provide a general setting to wavelets in Hilbert space.

Cardinal $B$-splines generate a large class of simple multiresolution approximations. This was extensively investigated by Chui and Wang [3, 4] who introduced the concept of duality. The object of this paper is to continue the investigation of wavelets associated with multiresolution approximations of multiplicity $r$. In $\S 2$ we give the general theory of wavelets which generate such multiresolution approximations, where we derive a general duality principle which is new even in the case $r=1$. Cardinal spline wavelets with multiple knots are introduced and studied in $\S 3$. These wavelets generate nonorthonormal Riesz bases for the multiresolution subspaces. Their basis properties are given in $\S 4$. Finally in $\S 5$, we consider the special cases $r=n+1$ and $n=2 r-1$, where $n$ is the degree of the spline functions, for which the wavelets can be constructed explicitly.

\section{WAVELETS WHICH GENERATE MULTIRESOLUTION APPROXIMATIONS OF MULTIPLICITY $r$}

For $\mathbf{s}=\left(s_{1}, \ldots, s_{r}\right) \in l^{2}(\mathbf{Z})^{r}$, its norm is given by

$$
\|\mathbf{s}\|:=\left(\sum_{j=1}^{r}\left\|s_{j}\right\|^{2}\right)^{1 / 2}
$$

The space of $2 \pi$-periodic square integrable functions and the space of $2 \pi$ periodic continuous functions will be denoted by $\widetilde{L}^{2}(0,2 \pi)$ and $\widetilde{C}(0,2 \pi)$ respectively. The Fourier transform of $f \in L^{2}(\mathbf{R})$ will be denoted by $\hat{f}$, and $f$ is said to be regular if $\hat{f}$ is continuous and $\hat{f}(u)=O\left(|u|^{-1}\right)$ as $|u| \rightarrow \infty$.

Let

such that

$$
\phi:=\left(\phi_{j}\right)_{j=1}^{r}, \quad \phi_{j} \in L^{2}(\mathbf{R}), \quad j=1, \ldots, r,
$$

$$
\sum_{n \in \mathbf{Z}} s(n) T_{n} \phi_{j} \in L^{2}(\mathbf{R}), \quad \forall s=(s(n))_{n \in \mathbf{Z}} \in l^{2}(\mathbf{Z})
$$

and let

$$
\boldsymbol{\psi}:=\left(\psi_{j}\right)_{j=1}^{r}, \quad \psi_{j} \in L^{2}(\mathbf{R}), \quad j=1, \ldots, r
$$


such that

$$
\sum_{n \in \mathbf{Z}} s(n) T_{n} \psi_{j} \in L^{2}(\mathbf{R}), \quad \forall s=(s(n))_{n \in \mathbf{Z}} \in l^{2}(\mathbf{Z}) .
$$

We shall assume throughout that $\phi_{j}$ and $\psi_{j}$ are regular for all $j=1, \ldots, r$. Let

$$
V_{0}:=\overline{\operatorname{span}\left\{T_{n} \phi_{j}: j=1,2, \ldots, r, n \in \mathbf{Z}\right\}}, \quad V_{1}:=\left\{D_{2} f: f \in V_{0}\right\}
$$

and assume that for $j=1,2, \ldots, r, \psi_{j} \in V_{1}$ and that $V_{0} \subset V_{1}$. Let $W_{0}$ be the orthogonal complement of $V_{0}$ in $V_{1}$. Then we have the 2-scale relations

$$
\phi(x)=2 \sum_{n \in \mathbf{Z}} \mathbf{P}_{n} \phi(2 x-n)
$$

and

$$
\boldsymbol{\psi}(x)=2 \sum_{n \in \mathbf{Z}} \mathbf{Q}_{n} \boldsymbol{\phi}(2 x-n),
$$

where $\left(\mathbf{P}_{n}\right)_{n \in \mathbf{Z}}$ and $\left(\mathbf{Q}_{n}\right)_{n \in \mathbf{Z}}$ are sequences of $r \times r$ matrices with entries in $l^{2}(\mathbf{Z})$.

Taking Fourier transform and letting

$$
\mathbf{P}(u)=\sum_{\nu \in \mathbf{Z}} \mathbf{P}_{\nu} e^{i \nu u} \quad \text { and } \quad \mathbf{Q}(u)=\sum_{\nu \in \mathbf{Z}} \mathbf{Q}_{\nu} e^{i \nu u},
$$

we have relations

$$
\hat{\phi}(2 u)=\mathbf{P}(u) \hat{\phi}(u)
$$

and

$$
\hat{\boldsymbol{\psi}}(2 u)=\mathbf{Q}(u) \hat{\boldsymbol{\phi}}(u),
$$

where $\mathbf{P}, \mathbf{Q}$ are $r \times r$ matrices with entries in $\widetilde{L}^{2}(0,2 \pi)$. We shall denote the set of all $r \times r$ matrices with entries in $\widetilde{L}^{2}(0,2 \pi)$ by $\widetilde{L}_{r \times r}^{2}(0,2 \pi)$, and the set of all $r \times r$ matrices with entries in $\widetilde{C}(0,2 \pi)$ by $\widetilde{C}_{r \times r}(0,2 \pi)$.

It follows immediately from the regularity of $\phi_{j}$ and $\psi_{j}$ that for all $u \in \mathbf{R}$, $\left(\hat{\phi}_{j}(u+2 \pi n)\right)_{n \in \mathbf{Z}}$ and $\left(\hat{\psi}_{j}(u+2 \pi n)\right)_{n \in \mathbf{Z}}$ belong to $l^{2}(\mathbf{Z})$. For $j, k=1, \ldots, r$ let

$$
\begin{aligned}
\Phi_{j k}(u) & :=\sum_{n \in \mathbf{Z}} \hat{\phi}_{j}(u+2 \pi n) \overline{\hat{\phi}_{k}(u+2 \pi n)} \\
\Psi_{j k}(u) & :=\sum_{n \in \mathbf{Z}} \hat{\psi}_{j}(u+2 \pi n) \overline{\hat{\psi}_{k}(u+2 \pi n)}
\end{aligned}
$$

and

$$
\Omega_{j k}(u):=\sum_{n \in \mathbf{Z}} \hat{\phi}_{j}(u+2 \pi n) \overline{\hat{\psi}_{k}(u+2 \pi n)} .
$$

Furthermore $\Phi_{j k}, \Psi_{j k}$ and $\Omega_{j k}$ belong to $\widetilde{C}(0,2 \pi)$. Let $\boldsymbol{\Phi}=\left(\Phi_{j k}\right)_{j, k=1}^{r}$, $\Psi=\left(\Psi_{j k}\right)_{j, k=1}^{r}$ and $\Omega=\left(\Omega_{j k}\right)_{j, k=1}^{r}$. Clearly $\Phi$ and $\Psi$ are Hermitian matrices. Therefore,

$$
\mathbf{M}:=\left(\begin{array}{cc}
\Phi & \Omega \\
\Omega^{*} & \Psi
\end{array}\right)
$$

is also Hermitian. Their eigenvalues are nonnegative and they are invertible if and only if the eigenvalues are bounded away from zero (see [6]). The following results are reminiscent of Theorem 3.1 in [6]. 
Proposition 2.1. The set $\left\{T_{n} \phi_{j}: n \in \mathbf{Z}, j=1, \ldots, r\right\}$ is a Riesz basis for a subspace of $L^{2}(\mathbf{R})$ if and only if $\Phi$ is invertible, $\left\{T_{n} \psi_{j}: n \in \mathbf{Z}, j=1, \ldots, r\right\}$ is a Riesz basis for a subspace of $L^{2}(\mathbf{R})$ if and only if $\Psi$ is invertible, and $\left\{T_{n} \phi_{j}, T_{n} \psi_{j}: n \in \mathbf{Z}, j=1, \ldots, r\right\}$ is a Riesz basis for a subspace of $L^{2}(\mathbf{R})$ if and only if $\mathbf{M}$ is invertible.

The proposition can be proved in the manner of Theorem 3.1 in [6].

\section{Lemma 2.1. For $u \in \mathbf{R}$}

$$
\mathbf{M}(2 u)=\left(\begin{array}{ll}
\mathbf{P}(u) & \mathbf{P}(u+\pi) \\
\mathbf{Q}(u) & \mathbf{Q}(u+\pi)
\end{array}\right)\left(\begin{array}{cc}
\Phi(u) & 0 \\
0 & \Phi(u+\pi)
\end{array}\right)\left(\begin{array}{ll}
\mathbf{P}(u) & \mathbf{P}(u+\pi) \\
\mathbf{Q}(u) & \mathbf{Q}(u+\pi)
\end{array}\right)^{*} .
$$

Proof. Let $\mathbf{P}(u)=\left(p_{j k}(u)\right)_{j, k=1}^{r}$ and $\mathbf{Q}(u)=\left(q_{j k}(u)\right)_{j, k=1}^{r}$. The relations (2.3) and (2.4) give

$$
\hat{\phi}_{j}(2 u)=\sum_{l=1}^{r} p_{j l}(u) \hat{\phi}_{l}(u)
$$

and

$$
\hat{\psi}_{j}(2 u)=\sum_{l=1}^{r} q_{j l}(u) \hat{\phi}_{l}(u) .
$$

Therefore, the entries of the matrix $\Phi(u)$ can be expressed as

$$
\boldsymbol{\Phi}_{j k}(2 u)=\sum_{l=1}^{r} \sum_{m=1}^{r}\left\{p_{j l}(u) \Phi_{l m}(u) \overline{p_{k m}(u)}+p_{j l}(u+\pi) \Phi_{l m}(u+\pi) \overline{p_{k m}(u+\pi)}\right\}
$$

which leads to the relation

$$
\Phi(2 u)=\mathbf{P}(u) \Phi(u) \mathbf{P}(u)^{*}+\mathbf{P}(u+\pi) \Phi(u+\pi) \mathbf{P}(u+\pi)^{*} .
$$

Similarly

$$
\Psi(2 u)=\mathbf{Q}(u) \Phi(u) \mathbf{Q}(u)^{*}+\mathbf{Q}(u+\pi) \Phi(u+\pi) \mathbf{Q}(u+\pi)^{*}
$$

and

$$
\Omega(2 u)=\mathbf{P}(u) \Phi(u) \mathbf{Q}(u)^{*}+\mathbf{P}(u+\pi) \Phi(u+\pi) \mathbf{Q}(u+\pi)^{*} .
$$

Equations (2.6), (2.7) and (2.8) are equivalent to (2.5).

Theorem 2.1. The following are equivalent:

$$
\left\{T_{n} \phi_{j}, T_{n} \psi_{j}: n \in \mathbf{Z}, j=1,2, \ldots, r\right\} \text { forms a Riesz basis for } V_{1}
$$

$$
\left(\begin{array}{ll}
\mathbf{P}(u) & \mathbf{P}(u+\pi) \\
\mathbf{Q}(u) & \mathbf{Q}(u+\pi)
\end{array}\right) \text { and } \Phi(u) \text { are invertible. }
$$

If these holds, then for any sequences of $r \times r$ matrices $\left(\mathbf{A}_{n}\right)_{n \in \mathbf{Z}},\left(\mathbf{B}_{n}\right)_{n \in \mathbf{Z}}$ and $\left(\mathbf{C}_{n}\right)_{n \in \mathbf{Z}}$ with $\mathbf{A}(u):=\sum_{n \in Z} \mathbf{A}_{n} e^{-i n u}, \mathbf{B}(u):=\sum_{n \in Z} \mathbf{B}_{n} e^{-i n u}$ and $\mathbf{C}(u):=$ $\sum_{n \in Z} \mathbf{C}_{n} e^{-i n u}$ in $\widetilde{L}_{r \times r}^{2}(0, \pi)$,

$$
\sum_{n \in Z} \mathbf{C}_{n} \phi(2 x-n)=\sum_{n \in Z} \mathbf{A}_{n} \phi(x-n)+\sum_{n \in Z} \mathbf{B}_{n} \boldsymbol{\psi}(x-n)
$$

if and only if

$$
\frac{1}{2}(\mathbf{C}(u) \quad \mathbf{C}(u+\pi))=\left(\begin{array}{ll}
\mathbf{A}(2 u) & \mathbf{B}(2 u))
\end{array}\left(\begin{array}{ll}
\mathbf{P}(u) & \mathbf{P}(u+\pi) \\
\mathbf{Q}(u) & \mathbf{Q}(u+\pi)
\end{array}\right) .\right.
$$


Moreover if (2.9) holds, then $\Phi(u)$ and $\Psi(u)$ are positive definite and have inverses in $\tilde{C}_{r \times r}(0,2 \pi)$.

The functions $\psi_{j}, j=1,2, \ldots, r$, belong to $W_{0}$ if and only if

$$
\mathbf{P}(u) \Phi(u) \mathbf{Q}(u)^{*}+\mathbf{P}(u+\pi) \Phi(u+\pi) \mathbf{Q}(u+\pi)^{*}=0 .
$$

Proof. If (2.9) holds then (2.10) follows from Proposition 2.1 and Lemma 2.1. Conversely if (2.10) holds, then $\left\{T_{n} \phi_{j}, T_{n} \psi_{j}: n \in \mathbf{Z}, j=1, \ldots, r\right\}$ is a Riesz basis for a subspace of $L^{2}(\mathbf{R})$. A similar argument as in the proof of Theorem 3.5 in [6] shows that it is complete in $V_{1}$.

Suppose $\left\{T_{n} \phi_{j}, T_{n} \psi_{j}: n \in \mathbf{Z}, j=1, \ldots, r\right\}$ is a Riesz basis for $V_{1}$. Then (2.11) holds if and only if

$$
\frac{1}{2} \mathbf{C}(u) \hat{\boldsymbol{\phi}}(u)=\mathbf{A}(2 u) \hat{\boldsymbol{\phi}}(2 u)+\mathbf{B}(2 u) \hat{\boldsymbol{\psi}}(2 u) .
$$

This equation can be written as

$$
\frac{1}{2} \mathbf{C}(u) \hat{\boldsymbol{\phi}}(u)=\mathbf{A}(2 u) \mathbf{P}(u) \hat{\boldsymbol{\phi}}(u)+\mathbf{B}(2 u) \mathbf{Q}(u) \hat{\boldsymbol{\phi}}(u)
$$

by (2.3) and (2.4). Since $\left\{T_{n} \phi_{j}: n \in \mathbf{Z}, j=1, \ldots, r\right\}$ is a Riesz basis for $V_{0}$, it follows that

$$
\frac{1}{2} \mathbf{C}(u)=\mathbf{A}(2 u) \mathbf{P}(u)+\mathbf{B}(2 u) \mathbf{Q}(u) .
$$

Translating equation (2.16) by $\pi$ leads to

$$
\frac{1}{2} \mathbf{C}(u+\pi)=\mathbf{A}(2 u) \mathbf{P}(u+\pi)+\mathbf{B}(2 u) \mathbf{Q}(u+\pi) .
$$

Equations (2.16) and (2.17) are equivalent to (2.12).

If (2.10) holds, then $\left\{T_{n} \phi_{j}: n \in \mathbf{Z}\right\}$ and $\left\{T_{n} \psi_{j}: n \in \mathbf{Z}\right\}$ are Riesz bases. Therefore $\Phi$ and $\Psi$ are invertible and their eigenvalues are positive.

Finally, $\psi_{j} \in W_{0}, j=1, \ldots, r$ if and only if

$$
\left\langle T_{n} \phi_{j}, \psi_{k}\right\rangle=0, \quad j, k=1, \ldots, r, n \in \mathbf{Z}
$$

if and only if $\Omega=0$ which is equivalent to (2.13) because of (2.8).

Now assume (2.9) holds. In (2.11) we choose $\left(\mathbf{G}_{n}\right)_{n \in \mathbf{Z}}$ and $\left(\mathbf{H}_{n}\right)_{n \in \mathbf{Z}}$ so that

$$
\phi(2 x-l)=\sum_{n \in \mathbf{Z}} \mathbf{G}_{2 n-l} \phi(x-n)+\sum_{n \in \mathbf{Z}} \mathbf{H}_{2 n-l} \boldsymbol{\psi}(x-n), \quad l \in \mathbf{Z} .
$$

Let

$$
\mathbf{G}(u)=\sum_{n \in \mathbf{Z}} \mathbf{G}_{n} e^{-i n u} \quad \text { and } \quad \mathbf{H}(u)=\sum_{n \in \mathbf{Z}} \mathbf{H}_{n} e^{-i n u} .
$$

For $l=0,1$ respectively, (2.12) gives

$$
\left(\begin{array}{ll}
\mathbf{I} & \mathbf{I}
\end{array}\right)=(\mathbf{G}(u)+\mathbf{G}(u+\pi) H(u)+\mathbf{H}(u+\pi))+\left(\begin{array}{cc}
\mathbf{P}(u) & \mathbf{P}(u+\pi) \\
\mathbf{Q}(u) & \mathbf{Q}(u+\pi)
\end{array}\right),
$$

and

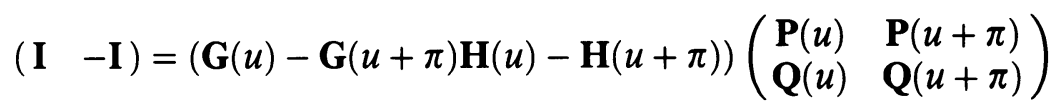

Adding these two equations gives

$$
\left(\begin{array}{ll}
\mathbf{I} & \mathbf{0}
\end{array}\right)=(\mathbf{G}(u) \mathbf{H}(u))\left(\begin{array}{ll}
\mathbf{P}(u) & \mathbf{P}(u+\pi) \\
\mathbf{Q}(u) & \mathbf{Q}(u+\pi)
\end{array}\right)
$$


or

$$
\mathbf{I}=\mathbf{G}(u) \mathbf{P}(u)+\mathbf{H}(u) \mathbf{Q}(u)
$$

and

$$
\mathbf{0}=\mathbf{G}(u) \mathbf{P}(u+\pi)+\mathbf{H}(u) \mathbf{Q}(u+\pi) .
$$

From (2.10) the solution to (2.20) and (2.21) for $\mathbf{G}$ and $\mathbf{H}$ is unique and we have seen that it gives (2.18) and (2.19). We shall derive formulae for $\mathbf{G}$ and $\mathbf{H}$ under assumption (2.13).

Theorem 2.2. If $\left\{T_{n} \phi_{j}: n \in \mathbf{Z}, j=1, \ldots, r\right\}$ and $\left\{T_{n} \psi_{j}: n \in \mathbf{Z}, j=\right.$ $1, \ldots, r\}$ form Riesz bases for $V_{0}$ and $W_{0}$ respectively, then $\mathbf{G}$ and $\mathbf{H}$ in (2.18), (2.19) are given by

$$
\mathbf{G}(u)=\Phi(u) \mathbf{P}(u)^{*} \Phi(2 u)^{-1}
$$

and

$$
\mathbf{H}(u)=\Phi(u) \mathbf{Q}(u)^{*} \Psi(2 u)^{-1} .
$$

Proof. Clearly (2.9) and (2.13) must hold. We thus have (2.20) and (2.21) which respectively give

$$
\mathbf{G}(u) \mathbf{P}(u) \Phi(u) \mathbf{P}(u)^{*}+\mathbf{H}(u) \mathbf{Q}(u) \Phi(u) \mathbf{P}(u)^{*}=\Phi(u) \mathbf{P}(u)^{*}
$$

and

(2.25) $\mathbf{G}(u) \mathbf{P}(u+\pi) \Phi(u+\pi) \mathbf{P}(u+\pi)^{*}+\mathbf{H}(u) \mathbf{Q}(u+\pi) \Phi(u+\pi) \mathbf{P}(u+\pi)^{*}=\mathbf{0}$

Adding and applying (2.6) and (2.13) leads to

$$
\mathbf{G}(u) \Phi(2 u)=\Phi(u) \mathbf{P}(u)^{*}
$$

which gives (2.22). Similarly (2.20) and (2.21) give

$$
\mathbf{G}(u) \mathbf{P}(u) \Phi(u) \mathbf{Q}(u)^{*}+\mathbf{H}(u) \mathbf{Q}(u) \Phi(u) \mathbf{Q}(u)^{*}=\Phi(u) \mathbf{Q}(u)^{*}
$$

and

(2.27) $\mathbf{G}(u) \mathbf{P}(u+\pi) \Phi(u+\pi) \mathbf{Q}(u+\pi)^{*}+\mathbf{H}(u) \mathbf{Q}(u+\pi) \Phi(u+\pi) \mathbf{Q}(u+\pi)^{*}=\mathbf{0}$

Adding and applying (2.7) and (2.13) leads to

$$
\mathbf{H}(u) \Psi(2 u)=\Phi(u) \mathbf{Q}(u)^{*}
$$

which gives $(2.23)$.

Assume that (2.9) hold and define $\tilde{\phi}=\left(\tilde{\phi}_{j}\right)_{j=1}^{r}, \tilde{\boldsymbol{\psi}}=\left(\tilde{\psi}_{j}\right)_{j=1}^{r}$, where $\tilde{\phi}_{j}$, $\tilde{\psi}_{j} \in V_{1}, j=1, \ldots, r$, by

$$
\hat{\tilde{\phi}}:=\Phi^{-1} \hat{\boldsymbol{\phi}}, \quad \hat{\tilde{\psi}}:=\Psi^{-1} \hat{\boldsymbol{\psi}} .
$$


Then

$$
\begin{aligned}
\left\langle\phi_{j}, T_{n} \tilde{\phi}_{k}\right\rangle & =\frac{1}{2 \pi} \int_{-\infty}^{\infty} \hat{\phi}_{j}(u) \overline{\hat{\tilde{\phi}}_{k}(u)} e^{i n u} d u \\
& =\frac{1}{2 \pi} \int_{-\infty}^{\infty} \hat{\phi}_{j}(u) \sum_{l=1}^{r} \overline{\Phi_{k l}^{-1}(u) \hat{\phi}_{l}(u)} e^{i n u} d u \\
& =\frac{1}{2 \pi} \sum_{\nu \in \mathbf{Z}} \int_{2 \pi \nu}^{2 \pi(\nu+1)} \hat{\phi}_{j}(u) \sum_{l=1}^{r} \Phi_{l k}^{-1}(u) \overline{\hat{\phi}_{l}(u)} e^{i n u} d u \\
& =\frac{1}{2 \pi} \sum_{\nu \in \mathbf{Z}} \int_{0}^{2 \pi} \hat{\phi}_{j}(u+2 \pi \nu) \sum_{l=1}^{r} \Phi_{l k}^{-1}(u) \overline{\hat{\phi}_{l}(u+2 \pi \nu)} e^{i n u} d u \\
& =\frac{1}{2 \pi} \int_{0}^{2 \pi} \sum_{l=1}^{r} \Phi_{j l}(u) \Phi_{l k}^{-1}(u) e^{i n u} d u \\
& =\frac{1}{2 \pi} \int_{0}^{2 \pi} \delta_{j, k} e^{i n u} d u=\delta_{j, k} \delta_{n, 0} .
\end{aligned}
$$

Thus $\tilde{\phi}$ is dual to $\phi$. Similarly $\tilde{\boldsymbol{\psi}}$ is dual to $\boldsymbol{\psi}$, i.e. $\left\langle\psi_{j}, T_{n} \tilde{\psi}_{k}\right\rangle=\delta_{j, k} \delta_{n, 0}$ for $j, k=1, \ldots, r, n \in \mathbf{Z}$.

Theorem 2.3. Under the assumption of Theorem 2.2, the dual functions $\tilde{\boldsymbol{\phi}}, \tilde{\boldsymbol{\psi}}$ satisfy the equations

$$
\begin{aligned}
\tilde{\boldsymbol{\phi}}(x) & =2 \sum_{n \in \mathbf{Z}} \mathbf{G}_{n}^{*} \tilde{\boldsymbol{\phi}}(2 x-n), \\
\tilde{\boldsymbol{\psi}}(x) & =2 \sum_{n \in \mathbf{Z}} \mathbf{H}_{n}^{*} \tilde{\boldsymbol{\phi}}(2 x-n)
\end{aligned}
$$

and

$$
\tilde{\boldsymbol{\phi}}(2 x-l)=\sum_{n \in \mathbf{Z}} \mathbf{P}_{2 n-l}^{*} \tilde{\boldsymbol{\phi}}(x-n)+\sum_{n \in \mathbf{Z}} \mathbf{Q}_{2 n-l}^{*} \tilde{\boldsymbol{\psi}}(x-n), \quad l \in \mathbf{Z} .
$$

Proof. By (2.3), (2.22) and (2.28)

$$
\begin{aligned}
\hat{\tilde{\phi}}(2 u) & =\Phi^{-1}(2 u) \hat{\phi}(2 u)=\Phi^{-1}(2 u) \mathbf{P}(u) \hat{\boldsymbol{\phi}}(u) \\
& =\Phi^{-1}(2 u) \mathbf{P}(u) \Phi(u) \hat{\tilde{\phi}}(u)=\mathbf{G}(u)^{*} \hat{\tilde{\phi}}(u) .
\end{aligned}
$$

This gives (2.29) by (2.19).

Similarly

$$
\begin{aligned}
\hat{\tilde{\boldsymbol{\psi}}}(2 u) & =\Psi^{-1}(2 u) \hat{\boldsymbol{\psi}}(2 u)=\Psi^{-1}(2 u) \mathbf{Q}(u) \hat{\boldsymbol{\phi}}(u) \\
& =\Psi^{-1}(2 u) \mathbf{Q}(u) \Phi(u) \tilde{\hat{\phi}}(u)=\mathbf{H}(u)^{*} \tilde{\hat{\phi}}(u)
\end{aligned}
$$

which gives (2.30). From (2.20) and (2.21) we have

$$
\mathbf{I}=\mathbf{P}(u)^{*} \mathbf{G}(u)^{*}+\mathbf{Q}(u)^{*} \mathbf{H}(u)^{*}
$$

and

$$
\mathbf{0}=\mathbf{P}(u)^{*} \mathbf{G}(u+\pi)^{*}+\mathbf{Q}(u)^{*} \mathbf{H}(u+\pi)^{*} .
$$

Thus the previous theory holds with $\mathbf{P}, \mathbf{Q}, \mathbf{G}, \mathbf{H}$ replaced respectively by $\mathbf{G}^{*}, \mathbf{H}^{*}, \mathbf{P}^{*}, \mathbf{Q}^{*}$, and corresponding to (2.18) we have (2.31). 
Thus the dual functions $\tilde{\phi}, \tilde{\psi}$ satisfy the same 2-scale relations (2.1) and (2.2) and the decomposition formula (2.18) as the functions $\phi, \psi$ with $\mathbf{P}$, $\mathbf{Q}, \mathbf{G}, \mathbf{H}$ replaced respectively by $\mathbf{G}^{*}, \mathbf{H}^{*}, \mathbf{P}^{*}, \mathbf{Q}^{*}$.

Remark 1 . The duality principle in this generality was not known before even for the case $r=1$. In [4] Chui and Wang assumed the sequence $\left(\mathbf{P}_{n}\right)_{n \in \mathbf{Z}}$ is finite and $\left(\mathbf{Q}_{n}\right)_{n \in \mathbf{Z}}$ has exponential decay as $|n| \rightarrow \infty$.

\section{SPLINE WAVELETS OF MULTIPLICITY $r$}

We denote by $\mathscr{S}_{n, r}(S)$ the space of spline functions of degree $n$ on $\mathbf{R}$ with knots of multiplicity $r$ on the set $S$. We write $V_{0}:=\mathscr{S}_{n, r}(\mathbf{Z}) \cap L^{2}(\mathbf{R})$ for some integers $n, r$, with $1 \leq r \leq n+1$. We let

$$
V_{1}:=\left\{f(2 x): f \in V_{0}\right\}, \quad \text { i.e. } \quad V_{1}=\mathscr{S}_{n, r}\left(\frac{1}{2} \mathbf{Z}\right) \cap L^{2}(\mathbf{R}),
$$

and let $W_{0}$ be the orthogonal complement of $V_{0}$ in $V_{1}$.

For $j \in \mathbf{Z}$ we define $t_{i}=j, j r \leq i \leq(j+1) r-1$. Thus the sequence $\left(t_{i}\right)_{-\infty}^{\infty}$ comprises integer knots with multiplicity $r$. We let $N_{i}^{n}$ denote the $B$-spline in $\mathscr{S}_{n, r}(\mathbf{Z})$ with support on $\left[t_{i}, t_{i+n+1}\right]$ and knots at $t_{i}, \ldots, t_{i+n+1}$, normalised so that $\sum_{i \in \mathbf{Z}} N_{i}^{n} \equiv 1$. It follows from the work of [1] that

$$
\left\{T_{j} N_{i}^{n}: j \in \mathbf{Z}, i=0, \ldots, r-1\right\}=\left\{N_{i}^{n}: i \in \mathbf{Z}\right\}
$$

forms a Riesz basis for $V_{0}$. We shall construct a corresponding Riesz basis

$$
\left\{T_{j} \psi_{i}: j \in \mathbf{Z}, i=0, \ldots, r-1\right\}=\left\{\psi_{i}: i \in \mathbf{Z}\right\}
$$

for $W_{0}$. By the integer knots of an element of $\mathscr{S}_{n, r}\left(\frac{1}{2} \mathbf{Z}\right)$ we shall mean those of its knots which are integers.

Theorem 3.1. For each $i \in \mathbf{Z}$, there is a nontrivial element $\psi_{i} \in W_{0}$, unique up to normalisation, with support on $\left[t_{i}, t_{i+2 n+2-r}\right]$ and integer knots at $t_{i}, \ldots$, $t_{i+2 n+2-r}$. The function $\psi_{i}$ does not have support on a smaller interval than $\left[t_{i}, t_{i+2 n+2-r}\right]$, neither does it have integer knots only at $t_{i}, \ldots, t_{i+2 n+1-r}$, nor integer knots only at $t_{i+1}, \ldots, t_{i+2 n+2-r}$.

We shall prove Theorem 3.1 by first constructing functions $\Psi_{i}$ in the space

$$
U:=\left\{f \in \mathscr{S}_{2 n+1, r}\left(\frac{1}{2} \mathbf{Z}\right): f^{(i)}(j)=0, j \in \mathbf{Z}, i=0, \ldots, r-1\right\} .
$$

The connection between $U$ and $W_{0}$ is given by

Lemma 3.1. If $f \in W_{0}$ has support on $[a, b]$, where $a<b$, then there is $a$ unique function $g \in U$ with support on $[a, b]$ and $g^{(n+1)}=f$. Conversely if $g \in U$ has support on $[a, b]$, then $g^{(n+1)}$ is in $W_{0}$.

Proof. The results follow easily using integration by parts.

Theorem 3.1 then follows immediately from Lemma 3.1 and

Theorem 3.2. For each $i \in \mathbf{Z}$, there is a nontrivial element $\Psi_{i} \in U$, unique up to normalisation, with support on $\left[t_{i}, t_{i+2 n+2-r}\right]$ and integer knots at $t_{i}, \ldots$, $t_{i+2 n+2-r}$. The function $\Psi_{i}$ does not have support on a smaller interval than $\left[t_{i}, \ldots, t_{i+2 n+2-r}\right]$, neither does it have integer knots only at $t_{i}, \ldots, t_{i+2 n+1-r}$, nor integer knots only at $t_{i+1}, \ldots, t_{i+2 n+2-r}$.

Proof. Without loss of generality we may assume $t_{i}=0$. We write $t_{i+2 n+2-r}=$ : $T$. Let $\mathscr{S}$ denote the space of functions in $\mathscr{S}_{2 n+1, r}\left(\frac{1}{2} \mathrm{Z}\right)$ with support in $[0, T]$ 
and integer knots at $t_{i}, \ldots, t_{i+2 n+2-r}$. Now any function in $\mathscr{S}$ has at most $2 n+3-r$ active integer knots and $T r$ other active knots and thus

$$
\operatorname{dim} \mathscr{S}=2 n+3-r+T r-(2 n+2)=(T-1) r+1 .
$$

Hence $\mathscr{S}$ is spanned by $N_{i+j}^{2 n+1}(2 x), j=0, \ldots,(T-1) r$. Let $y_{j}, j=$ $1, \ldots,(T-1) r$, be the integer knots in $(0, T)$, i.e. for $k=1, \ldots, N-1$,

$$
y_{j}=k, \quad(k-1) r+1 \leq j \leq k r .
$$

We shall show that for $j=1, \ldots,(T-1) r, y_{j}$ lies in the interior of the support of $N_{i+j}^{2 n+1}(2 x)$, i.e.

$$
\frac{1}{2} t_{i+j}<y_{j}<\frac{1}{2} t_{i+j+2 n+2} .
$$

Now

$$
t_{i+j}= \begin{cases}0, & j=0, \ldots, r-i-1, \\ 1, & j=r-i, \ldots, 2 r-i-1,\end{cases}
$$

and so

$$
\frac{1}{2} t_{i+j}<1=y_{j}, \quad j=1, \ldots, r .
$$

Since

$$
t_{i+j+r}=t_{i+j}+1, y_{j+r}=y_{j}+1, \quad j=1, \ldots,(T-2) r,
$$

we have

$$
\frac{1}{2} t_{i+j}<y_{j}, \quad j=1, \ldots,(T-1) r .
$$

Similarly

$$
\frac{1}{2} t_{i+j+2 n+2}>T-1=y_{j}, \quad j=(T-2) r+1, \ldots,(T-1) r,
$$

and from (3.2) we have

$$
\frac{1}{2} t_{i+j+2 n+2}>y_{j}, \quad j=1, \ldots,(T-1) r .
$$

Thus we have (3.1). By the Schoenberg-Whitney theorem [12], any function in $\operatorname{span}\left\{N_{i+j}^{2 n+1}: j=1, \ldots,(T-1) r\right\}$ which vanishes at $y_{j}, j=1, \ldots$, $(T-1) r$, must be identically zero, i.e. there is no nontrivial function in $U$ with support on $\left[\frac{1}{2} t_{i+1}, T\right]$ with knots at $\frac{1}{2} t_{i+j}, j=1, \ldots,(T-1) r+2 n+2$. Thus there is no nontrivial function in $U$ with support in $(0, T]$, neither is there a nontrivial function in $U$ with support on $[0, T]$ with integer knots only at $t_{i+1}, \ldots, t_{i+2 n+2-r}$.

Similarly there is no nontrivial function in $U$ with support in $[0, T)$, neither is there a nontrivial function with support in $[0, T]$ and integer knots only at $t_{i}, \ldots, t_{i+2 n+1-r}$.

It remains only to show that there is a nontrivial function in $U$, unique up to normalisation, with support on $[0, T]$ and integer knots at $t_{i}, \ldots, t_{i+2 n+2-r}$. But this follows from applying the Schoenberg-Whitney theorem to the space $\mathscr{S}$ and the points $y_{j}, j=0, \ldots,(T-1) r$, where we can take $y_{0}=1 / 2$.

The normalisation of $\Psi_{i}$ is unimportant, but we obviously choose it so that $\Psi_{i+r}=\Psi_{i}(\cdot-1), i \in \mathbf{Z}$. Putting $\psi_{i}=\Psi_{i}^{(n+1)}$ gives Theorem 3.1. 
Theorem 3.3. For $i \in \mathbf{Z}$, the function $\psi_{i}$ does not vanish on any nontrivial interval in $\left[t_{i}, t_{i+2 n+2-r}\right]$ and it has precisely $(T-1) r+n+1$ changes of sign, where $T=t_{i+2 n+2-r}-t_{i}$.

Proof. We assume $t_{i}=0$ and employ the notation of the proof of Theorem 3.2. First note that $\Psi_{i}$ does not vanish on any nontrivial interval in $[0, T]$, for if it did there would be a nontrivial function in $\mathscr{S} \cap U$ with support on a smaller interval than $[0, T]$, which contradicts Theorem 3.2.

Now by Rolle's theorem, $\Psi_{i}^{\prime}$ has at least $T$ changes of sign. In addition, $\Psi_{i}^{\prime}$ vanishes at 0 and $T$ and has zeros of multiplicity $r-1$ at $1, \ldots, T-1$, and is nonzero somewhere between $j$ and $j+1, j=0, \ldots, T-1$. Recursively we see that $\psi_{i}=\Psi_{i}^{(n+1)}$ has at least $(T-1) r+n+1$ changes of sign. Now $\psi_{i}$ lies in $\left\{f^{(n+1)}: f \in \mathscr{S}\right\}$, which is spanned by $N_{i+j}^{n}(2 x), j=0, \ldots,(T-1) r+n+1$. So by the variation diminishing property [2], $\psi_{i}$ has at most $(T-1) r+n+1$ changes of sign.

It remains to show that $\psi_{i}$ cannot vanish on any nontrivial interval in $[0, T]$. By Theorem 3.1, it cannot vanish on $\left(0, \frac{1}{2}\right)$ nor on $\left(T-\frac{1}{2}, T\right)$. Suppose it vanishes on some nontrivial interval $(a, b)$ in $\left(\frac{1}{2}, T-\frac{1}{2}\right)$. Then for some $l \geq 1,\left.\psi_{i}\right|_{[0, a]}$ is a combination of $N_{i+j}^{n}(2 x), j=0, \ldots, l-1$, and $\left.\psi_{i}\right|_{[b, T]}$ is a combination of $N_{i+j}^{n}(2 x), j=l+n+1, \ldots,(T-1) r+n+1$. So $\left.\psi_{i}\right|_{[0, a]}$ has at most $l-1$ changes of sign and $\left.\psi_{i}\right|_{[b, T]}$ has at most $(T-1) r-l$ changes of sign. So $\psi_{i}$ has at most $(T-1) r$ changes of sign, which is a contradiction.

\section{LINEAR COMBINATIONS OF WAVELETS}

We say a sequence $\left(f_{i}\right)_{i=-\infty}^{\infty}$ of functions is locally linearly independent on a nontrivial interval $(a, b)$ if whenever $\sum_{i \in \mathbf{Z}} c_{i} f_{i}$ vanishes identically on $(a, b)$, then $c_{i}=0$ for all $i$ for which $f_{i}$ does not vanish identically on $(a, b)$.

Theorem 4.1. The sequence $\left(\Psi_{i}\right)_{i=-\infty}^{\infty}$ is locally linearly independent on any interval.

Proof. Take $a<b$ and suppose $f=\sum_{i \in \mathbf{Z}} c_{i} \Psi_{i}$ vanishes identically on $(a, b)$. Suppose that $\Psi_{i}, j \leq i \leq k$, are those functions $\Psi_{i}$ whose supports overlap $(a, b)$, i.e. which do not vanish identically on $(a, b)$. We need to show $c_{i}=0$, $j \leq i \leq k$.

Let $S=\sum_{i=j}^{k} c_{i} \Psi_{i}$. Then for $a<t<b, S(t)=f(t)$ and so $S$ vanishes identically on $(a, b)$. Define $S_{1}$ in $U$ by

$$
S_{1}(t)= \begin{cases}S(t), & t \leq a \\ 0, & \text { otherwise }\end{cases}
$$

Then $S_{1}$ has only the same integer knots as $\Psi_{j}$ and smaller support. So by Theorem $3.2, S_{1} \equiv 0$, i.e. $S$ vanishes identically on $(-\infty, a]$. Similarly we see that $S$ vanishes identically on $[b, \infty)$ and thus $S \equiv 0$.

Now support that $t_{j}=\cdots=t_{j+s-1}=\alpha, t_{j+s}=\alpha+1$. Then for $i=j+$ $1, \ldots, k$, we have $\Psi_{i}^{(2 n+2-s)}(\alpha)=0$. But by Theorem 3.2, $\Psi_{j}^{(2 n+2-s)}\left(\alpha^{+}\right) \neq 0$. Since $0=S^{(2 n+2-s)}\left(\alpha^{+}\right)=c_{j} \Phi_{j}^{(2 n+2-s)}\left(\alpha^{+}\right)$, we must have $c_{j}=0$. Proceeding in this way we see that $c_{i}=0, j \leq i \leq k$. 
Corollary 4.1. Any function $f \in U$ can be written uniquely in the form

$$
f=\sum_{i \in \mathbf{Z}} c_{i} \Psi_{i}
$$

for some constants $c_{i}, i \in \mathbf{Z}$. Moreover there is a constant $K$ such that if the support of $\Psi_{i}$ overlaps the interval $(j, j+1)$, then $\left|c_{i}\right| \leq K\left\|\left.f\right|_{[j, j+1]}\right\|_{\infty}$, where $K$ is independent of $i, j$ and $f$.

Proof. Take any integers $j, M$ with $M \geq 1$ and let

$$
\mathscr{S}:=\left.\mathscr{S}_{2 n+1, r}\left(\frac{1}{2} \mathbf{Z}\right)\right|_{[j, j+M]}, \quad \mathscr{S}_{0}:=\left.U\right|_{[j, j+M]} .
$$

It is easily seen that $\operatorname{dim} \mathscr{S}=2 n+2+r(2 M-1)$ and that the Hermite interpolation problem for $\mathscr{S}$ with nodes of multiplicity $n+1$ at $j$ and $j+M$ and nodes of multiplicity $r$ on $\frac{1}{2} \mathrm{Z} \cap(j, j+M)$ is well-poised. Since $\mathscr{S}_{0}$ comprises elements of $\mathscr{S}$ with zeros of multiplicity $r$ at $j+i, i=0, \ldots, M$,

$$
\operatorname{dim} \mathscr{S}_{0}=2 n+2+r(2 M-1)-r(M+1)=2 n+2+r(M-2) .
$$

But the function $\Psi_{i}$ has support overlapping $(j, j+M)$ if and only if $t_{i}<j+M$ and $t_{i+2 n+2-r}>j$, i.e. $i<(j+M) r$ and $i+2 n+2-r \geq(j+1) r$, i.e. $(j+2) r-2 n-2 \leq i \leq(j+M) r-1$. So the number of such functions is $2 n+2+r(M-2)$ and by Theorem 4.1, these functions restricted to $(j, j+M)$ form a basis for $\mathscr{S}_{0}$.

By considering arbitrarily large intervals we see that any function $f \in U$ can be written uniquely in the form $f=\sum_{i \in \mathbf{Z}} c_{i} \Psi_{i}$.

Once again take an integer $j$. We have seen that $\left.\Psi_{i}\right|_{[j, j+1]},(j+2) r-2 n-2 \leq$ $i \leq(j+1) r-1$, form a basis for $\left.U\right|_{[j, j+1]}$. Since norms on a finite dimensional space are equivalent, there is a constant $K$ such that for all $f \in U$,

$$
\max \left\{\left|c_{i}\right|:(j+2) r-2 n-2 \leq i \leq(j+1) r-1\right\} \leq K\left\|\left.f\right|_{[j, j+1]}\right\|_{\infty} .
$$

Since $K$ is clearly independent of $j$, this completes the proof.

We now consider corresponding results for the wavelets $\psi_{i}$.

Theorem 4.2. The sequence $\left(\psi_{i}\right)_{i=-\infty}^{\infty}$ is locally linearly independent on any interval $(j, j+M)$ for any integers $j, M$ with $r(M+1) \geq n+1$.

Proof. Suppose $\sum_{i \in \mathbf{Z}} c_{i} \psi_{i}=0$. Without loss of generality we assume $j=$ 0 . As in the proof of Corollary 4.1, the function $\psi_{i}$ has support overlapping $(0, M)$ if and only if $2 r-2 n-2 \leq i \leq M r-1$. Let $f=\sum_{i=2 r-2 n-2}^{M r-1} c_{i} \psi_{i}$. Then $f$ vanishes on $(0, M)$. So $f=g+h$, where $g$ has support on $\left[t_{2 r-2 n-2}, 0\right]$ and $h$ has support on $\left[M, t_{(M-1) r+2 n+1}\right]$. Now suppose $i \leq-1$. Then $t_{i+n+1} \leq$ $t_{n} \leq t_{r(M+1)-1}=M$, and so the support of $N_{i}^{n}$ does not overlap the support of $h$. Thus $\int_{-\infty}^{\infty} N_{i}^{n} h=0$ and since $f$ is in $W_{0}$,

$$
\int_{-\infty}^{\infty} N_{i}^{n} g=\int_{-\infty}^{\infty} N_{i}^{n} f=0 .
$$

Next suppose $i \geq 0$. Then $t_{i} \geq t_{0}=0$ and so the support of $N_{i}^{n}$ does not overlap the support of $g$. Thus $\int_{-\infty}^{\infty} N_{i}^{n} g=0$ and so

$$
\int_{-\infty}^{\infty} N_{i}^{n} h=\int_{-\infty}^{\infty} N_{i}^{n} f=0 .
$$


So both $g$ and $h$ lie in $W_{0}$. But the support of $g$ is smaller than that of $\psi_{2 r-2 n-2}$ and so $g \equiv 0$ by Theorem 3.1. Similarly $h \equiv 0$ and so $f \equiv 0$.

As in the last part of the proof of Theorem 4.1, we can show successively that $c_{i}=0,2 r-2 n-2 \leq i \leq M r-1$.

Corollary 4.2. For integers $j, M$ with $M \geq 1$,

$$
\begin{aligned}
\left.W_{0}\right|_{[j, j+M]}=\left\{\left.f \in \mathscr{S}_{n, r}\left(\frac{1}{2} \mathbf{Z}\right)\right|_{[j, j+M]}: \int_{-\infty}^{\infty} f g\right. & =0 \text { for all } g \in \mathscr{S}_{n, r}(\mathbf{Z}) \\
& \text { with support in }[j, j+M]\} .
\end{aligned}
$$

Proof. Again we may assume $j=0$. Let $\mathscr{X}:=\left.\mathscr{S}_{n, r}\left(\frac{1}{2} \mathbf{Z}\right)\right|_{[0, M]}$ and

$$
\mathscr{Y}:=\left\{f \in \mathscr{X}: \int_{-\infty}^{\infty} f g=0 \text { for all } g \in \mathscr{S}_{n, r}(\mathbf{Z}) \text { with support in }[0, M]\right\} .
$$

First suppose $r(M+1) \geq n+1$. Then

$$
\mathscr{Y}=\left\{f \in \mathscr{X}: \int_{-\infty}^{\infty} f N_{i}^{n}=0, i=0, \ldots,(M+1) r-n-2\right\} .
$$

Now the linear functionals on $\mathscr{X}, f \mapsto \int_{-\infty}^{\infty} f N_{i}^{n}, i=0, \ldots,(M+1) r-n-$ $2=: L$, are linearly independent, for if

$$
\int_{-\infty}^{\infty} f \sum_{i=0}^{L} c_{i} N_{i}^{n}=0 \text { for all } f \text { in } \mathscr{Z},
$$

then

$$
\int_{-\infty}^{\infty}\left(\sum_{i=0}^{L} c_{i} N_{i}\right)^{2}=0 \text { and } c_{i}=0, \quad i=0, \ldots, L
$$

Thus

$$
\begin{aligned}
\operatorname{dim} \mathscr{Y} & =\operatorname{dim} \mathscr{X}-\{(M+1) r-n-1\} \\
& =n+1+(2 M-1) r-(M+1) r+n+1 \\
& =2 n+2+r(M-2) .
\end{aligned}
$$

So from Theorem 4.2 and its proof, $\operatorname{dim}\left(\left.W_{0}\right|_{[0, M]}\right) \geq \operatorname{dim} \mathscr{Y}$. But clearly $\left.W_{0}\right|_{[0, M]} \subset \mathscr{Y}$ and so $\left.W_{0}\right|_{[0, M]}=\mathscr{Y}$.

Now suppose $r(M+1) \leq n+1$. Since $\left.W_{0}\right|_{[0, M]} \subset \mathscr{Y}=\mathscr{X}$, we must show $\left.\mathscr{Z} \subset W_{0}\right|_{[0, M]}$. Take $f \in \mathscr{Z}$ and $\left.g \in \mathscr{S}_{2 n+1, r}\left(\frac{1}{2} \mathbf{Z}\right)\right|_{[0, M]}$ with $g^{(n+1)}=f$. Since $r(M+1) \leq n+1$, we may choose $p \in \Pi_{n}$, polynomials of degree $n$, so that $g-p$ satisfies

$$
(g-p)^{(j)}(k)=0, \quad j=0, \ldots, r-1, k=0, \ldots, M .
$$

Thus $g-p$ lies in $\left.U\right|_{[0, M]}$ and extending $g-p$ to an element of $U$ with compact support, we can apply Lemma 3.1 to show that $f=(g-p)^{(n+1)}$ lies in $\left.W_{0}\right|_{[0, M]}$.

We remark that Theorem 4.2 and Corollary 4.2 have not appeared before even for the case $r=1$. 
Corollary 4.3. Any function in $W_{0}$ can be written uniquely in the form $\sum_{i \in \mathbf{Z}} c_{i} \psi_{i}$ for some constants $c_{i}, i \in \mathbf{Z}$.

Proof. Take $f \in W_{0}$ and any integer $M$ with $r(M+1) \geq n+1$. As in the proof of Corollary 4.2 we can write

$$
\left.f\right|_{[0, M]}=\left.\sum_{i=2 r-2 n-2}^{M r-1} c_{i} \psi_{i}\right|_{[0, M]}
$$

for some constants $c_{i}, i \in \mathbf{Z}$, and by Theorem 4.2, these constants are unique. The corresponding result clearly applies on any interval $[j, j+M]$ for any integer $j$, and considering arbitrarily large intervals gives the result.

We remark that in fact we have proved the following stronger result.

Any function $f$ in $\mathscr{S}_{n, r}\left(\frac{1}{2} \mathbf{Z}\right)$ which satisfies $\int_{-\infty}^{\infty} f N_{i}^{n}=0$, for all $i \in \mathbf{Z}$, can be written uniquely in the form $f=\sum_{i \in \mathbf{Z}} c_{i} \psi_{i}$ for some constants $c_{i}, i \in \mathbf{Z}$.

Corollary 4.4. The functions $\left(\psi_{i}\right)_{i=-\infty}^{\infty}$ form a Riesz basis for $W_{0}$.

Proof. Take $M$ so that $r(M+1) \geq n+1$. Take $f=\sum_{i \in \mathbf{Z}} c_{i} \psi_{i}$ in $W_{0}$. For any integer $j,\left.\psi_{i}\right|_{[j, j+M]}, l:=(j+2) r-2 n-2 \leq i \leq(j+M) r-1=: L$, form a basis for $\left.W_{0}\right|_{[j, j+M]}$ and so there are constants $A, B$, independent of $f$ and $j$, so that

$$
A \int_{j}^{j+M} f^{2} \leq \sum_{i=l}^{L} c_{i}^{2} \leq B \int_{j}^{j+M} f^{2} .
$$

Adding over $j$ gives

$$
M A\|f\|_{2}^{2} \leq(L-l+1) \sum_{i \in \mathbf{Z}} c_{i}^{2} \leq M B\|f\|_{2}^{2} .
$$

It follows from Corollary 4.4 that any function $f \in V_{1}$ can be written uniquely in the form

$$
f=\sum_{j \in \mathbf{Z}} a_{j} \psi_{j}+\sum_{j \in \mathbf{Z}} b_{j} N_{j}^{n},
$$

for sequences $\left(a_{j}\right)_{j=-\infty}^{\infty},\left(b_{j}\right)_{j=-\infty}^{\infty}$ in $l^{2}(\mathbf{Z})$. The following result can be deduced from the general theory of $\S 2$, but the proof below shows the connection with Schoenberg's theory of cardinal Hermite spline interpolation [11].

Theorem 4.3. If $f \in V_{1}$ has compact support, then $\left(a_{j}\right)_{j=-\infty}^{\infty}$ and $\left(b_{j}\right)_{j=-\infty}^{\infty}$ in (4.1) decay exponentially as $|j| \rightarrow \infty$.

Proof. Suppose that $f$ has support on $[a, b]$. Let $F$ be the function in $\mathscr{S}_{2 n+1, r}\left(\frac{1}{2} \mathbf{Z}\right)$ which vanishes on $(-\infty, a)$ and satisfies $F^{(n+1)}=f$. Then $\left.F\right|_{(b, \infty)}$ is in $\Pi_{n}$. By Schoenberg's theory [11], there is a unique element $S$ of $\mathscr{S}_{2 n+1, r}(\mathbf{Z})$ which interpolates $F$ with multiplicity $r$ on $\mathbf{Z}$. Since $F-S$ is in $\mathscr{S}_{2 n+1, r}\left(\frac{1}{2} \mathbf{Z}\right)$ and has zeros of multiplicity $r$ on $\mathbf{Z}$, we have $F=\Psi+S$ for some $\Psi \in U$. So by Corollary 4.1 , we can write uniquely

$$
F=\sum_{j \in \mathbf{Z}} a_{j} \Psi_{j}+\sum_{j \in \mathbf{Z}} c_{j} N_{j}^{2 n+1},
$$


for constants $a_{j}, c_{j}, j \in \mathbf{Z}$. Since $F=0$ and $(-\infty, a)$, Schoenberg's theory shows that $S(x)$ decays exponentially as $x \rightarrow-\infty$ and hence $\left(c_{j}\right)_{j=-\infty}^{\infty}$ decays exponentially as $j \rightarrow-\infty$. Differentiating $n+1$ time gives

$$
f=\sum_{j \in \mathbf{Z}} a_{j} \psi_{j}+\sum_{j \in \mathbf{Z}} b_{j} N_{j}^{n},
$$

where $\left(b_{j}\right)_{j=-\infty}^{\infty}$ decays exponentially as $j \rightarrow-\infty$.

Now choose $p \in \Pi_{n}$ with $F=p$ on $(b, \infty)$. Then $S-p$ interpolates $F-p$ with multiplicity $r$ on $\mathbf{Z}$ and since $F-p$ vanishes on $(b, \infty)$, we have $S(x)-p(x)$ decays exponentially as $x \rightarrow \infty$. Since

$$
\sum_{j \in \mathbf{Z}} b_{j} N_{j}=S^{(n+1)}=(S-p)^{(n+1)},
$$

we have $\left(b_{j}\right)_{j=-\infty}^{\infty}$ decays exponentially as $j \rightarrow \infty$.

Now $\Psi=F-S=-S$ on $(-\infty, a)$ and so $\Psi(x)$ decays exponentially as $x \rightarrow-\infty$. Furthermore on $(b, \infty), \Psi=F-S=p-S$ and so $\Psi(x)$ decays exponentially as $x \rightarrow \infty$. It follows from the last part of Corollary 4.1 that $\left(a_{j}\right)_{j=-\infty}^{\infty}$ decays exponentially as $|j| \rightarrow \infty$.

\section{CONSTRUCTION OF WAVELETS}

We now consider how to construct the wavelets $\left(\psi_{i}\right)_{i=-\infty}^{\infty}$. Since $\psi_{i+r}(x)=$ $\psi_{i}(x-1)$ for all $i \in \mathbf{Z}$, it is sufficient to consider $\psi_{0}, \ldots, \psi_{r-1}$.

Take $0 \leq i \leq r-1$ and suppose $\Psi_{i}$ has support on $[0, T]$. Then

$$
\Psi_{i}(x)=\sum_{j=0}^{(T-1) r} c_{j} N_{i+j}^{2 n+1}(2 x),
$$

where $c_{j}, j=0, \ldots,(T-1) r$, can be calculated from the relations

$$
\Psi_{i}^{(j)}(k)=0, \quad j=0, \ldots, r-1, k=1, \ldots, T-1,
$$

and some normalisation condition. Applying the differentiation recurrence relation for $B$-splines, we can then write

$$
\psi_{i}(x)=\Psi_{i}^{(n+1)}(x)=\sum_{j=0}^{(T-1) r+n+1} d_{j} N_{i+j}^{n}(2 x),
$$

for some constants $d_{j}, j=0, \ldots,(T-1) r+n+1$.

Henceforward we shall consider special cases for which we can derive more direct constructions. For the case $r=1$, there was given in [4] the elegant formula

$$
\Psi_{0}(x)=\sum_{j=0}^{2 n}(-1)^{j} N_{0}^{n}(j+1) N_{0}^{2 n+1}(2 x-j) .
$$

To see why this is so we write

$$
\Pi(z)=\sum_{\nu \in \mathbf{Z}} N_{0}^{n}(\nu) z^{\nu-1}
$$


and note that for any integer $k, \Psi_{0}(k)$ from (5.3) is the coefficient of $z^{2 k-1}$ in the even polynomial $\Pi(-z) \Pi(z)$ and hence $\Psi_{0}(k)=0$.

Unfortunately no corresponding formula appears to hold for $r \geq 2$. The next case we consider is $r=n+1$. Here $T=1,(5.2)$ becomes vacuous and (5.1) can be written

$$
\Psi_{i}(x)=N_{i}^{2 n+1}(2 x), \quad i=0, \ldots, n .
$$

Applying the differentiation recurrence relation gives, after some calculation,

$$
\psi_{i}(x)=\sum_{j=i}^{n} a_{i, j} N_{j}^{n}(2 x)-\sum_{j=0}^{i} a_{n-i, n-j} N_{j}^{n}(2 x-1), \quad i=0, \ldots, n,
$$

where

$$
a_{i, j}=(-1)^{j} \sum_{l=i}^{j} 2^{n-l}\left(\begin{array}{l}
l \\
i
\end{array}\right)\left(\begin{array}{l}
n-l \\
n-j
\end{array}\right) .
$$

Here the $B$-spline $N_{j}^{n}$ is given by

$$
N_{j}^{n}(x)= \begin{cases}\left(\begin{array}{l}
n \\
j
\end{array}\right) x^{j}(1-x)^{n-j} & \text { if } 0 \leq x<1 \\
0 & \text { elsewhere }\end{cases}
$$

Note that $\psi_{i}(x)=-\psi_{n-i}(1-x), i=0, \ldots, n$. Also $\psi_{i}$ has zeros of multiplicity $i$ at 0 and of multiplicity $n-i$ at 1 and so by Theorem $3.3 \psi_{i}$ changes sign at $\frac{1}{2}, n-i$ times in $\left(0, \frac{1}{2}\right)$ and $i$ times in $\left(\frac{1}{2}, 1\right)$.

Now given coefficients $c_{0}, \ldots, c_{n}$, we see from (5.4) that

$$
\sum_{i=0}^{n} c_{i} \psi_{i}(x)=\sum_{j=0}^{n}(-1)^{j} d_{j} N_{j}^{n}(2 x)+\sum_{j=0}^{n}(-1)^{n+j+1} e_{j} N_{j}^{n}(2 x-1),
$$

where for $j=0, \ldots, n$,

$$
\begin{aligned}
d_{j} & =\sum_{l=0}^{j} 2^{n-l}\left(\begin{array}{c}
n-l \\
n-j
\end{array}\right) \sum_{i=0}^{l} c_{i}\left(\begin{array}{l}
l \\
i
\end{array}\right), \\
e_{j} & =\sum_{l=0}^{n-j} 2^{n-l}\left(\begin{array}{c}
n-l \\
j
\end{array}\right) \sum_{i=0}^{l} c_{n-i}\left(\begin{array}{l}
l \\
i
\end{array}\right) .
\end{aligned}
$$

The coefficients $d_{j}, e_{j}, j=0, \ldots, n$, can be calculated from $c_{0}, \ldots, c_{n}$ by an algorithm which is illustrated schematically for $n=2$ in Figure 1 and for general $n$ in Figure 2. Here

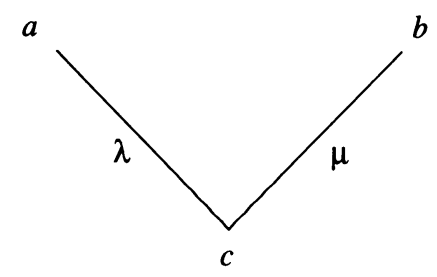

denotes $c=\lambda a+\mu b$. This algorithm is easily reversed so that if the coefficients $d_{0}, \ldots, d_{n}$ or $e_{0}, \ldots, e_{n}$ are known then $c_{0}, \ldots, c_{n}$ can be computed. 


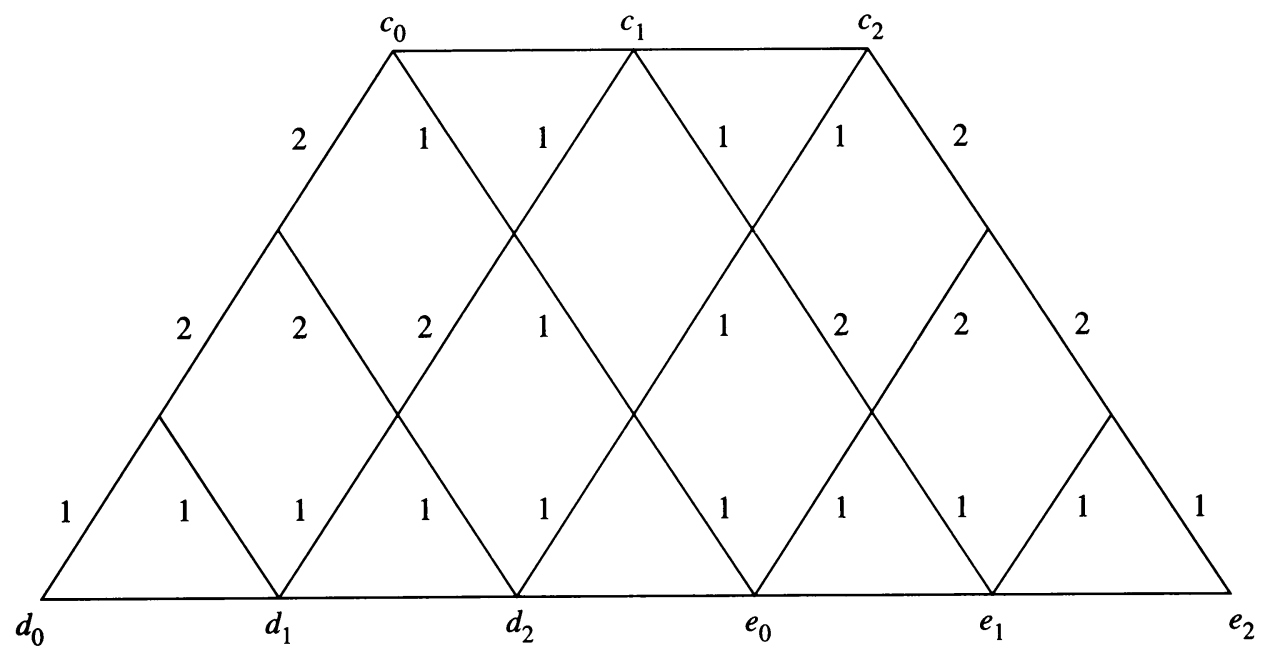

FIGURE 1

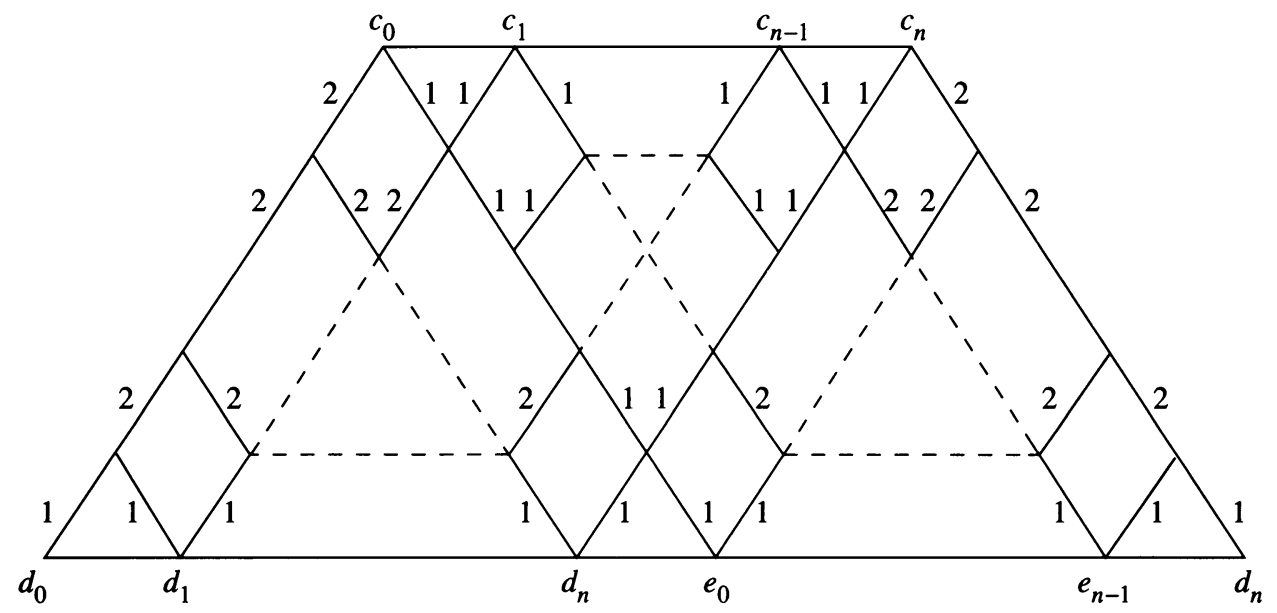

FIGURE 2

For the rest of this section we consider the case $n=2 r-1$. In this case $\Psi_{0}, \ldots, \Psi_{r-1}$ all have support on [0,3]. For computational convenience we shall construct a different basis for $\operatorname{span}\left\{\Psi_{0}, \ldots, \Psi_{r-1}\right\}$ comprising functions $F_{i}, i=1, \ldots,\left\lceil\frac{r}{2}\right\rceil, G_{i}, i=1, \ldots,\left\lfloor\frac{r}{2}\right\rfloor$, where the functions $F_{i}$ and $G_{i}$ are respectively even and odd about $\frac{3}{2}$. These are constructed recursively as follows. Let

$$
\begin{array}{ll}
F_{i, 0}(x)=N_{i-1}^{2 n+1}(2 x)+N_{3 r-i}^{2 n+1}(2 x), & i=1, \ldots,\lceil 3 r / 2\rceil, \\
G_{i, 0}(x)=N_{i-1}^{2 n+1}(2 x)-N_{3 r-i}^{2 n+1}(2 x), & i=1, \ldots,\lfloor 3 r / 2\rfloor,
\end{array}
$$

and for $j=1, \ldots, r$,

$$
\begin{gathered}
F_{i, j}(x)=F_{i, j-1}(x) F_{i+1, j-1}^{(j-1)}(1)-F_{i+1, j-1}(x) F_{i, j-1}^{(j-1)}(1), \\
i=1, \ldots,\lceil 3 r / 2\rceil-j, \\
G_{i, j}(x)=G_{i, j-1}(x) G_{i+1, j-1}^{(j-1)}(1)-G_{i+1, j-1}(x) G(j-1)_{i, j-1}(1), \\
i=1, \ldots,\lfloor 3 r / 2\rfloor-j .
\end{gathered}
$$


Then we have

$$
\begin{aligned}
& F_{i}=F_{i, r}, \quad i=1, \ldots,\lceil r / 2\rceil, \\
& G_{i}=G_{i, r}, \quad i=1, \ldots,\lfloor r / 2\rfloor .
\end{aligned}
$$

Lemam 5.1. The functions $F_{i}, i=1, \ldots,\left\lceil\frac{r}{2}\right\rceil, G_{i}, i=1, \ldots,\left\lfloor\frac{r}{2}\right\rfloor$, form $a$ basis for $\operatorname{span}\left\{\Psi_{0}, \ldots, \Psi_{r-1}\right\}$.

Proof. It is clear by induction that for $j=0, \ldots, r, F_{i, j}, i=1, \ldots,\left\lceil\frac{3 r}{2}\right\rceil-j$, are even about $\frac{3}{2}$ and $G_{i, j}, i=1, \ldots,\left\lfloor\frac{3 r}{2}\right\rfloor-j$, are odd about $\frac{3}{2}$. From (5.7) and (5.8) we have for $j=1, \ldots, r$,

$$
F_{i, j}^{(j-1)}(1)=G_{i, j}^{(j-1)}(1)=0,
$$

and by symmetry,

$$
F_{i, j}^{(j-1)}(2)=G_{i, j}^{(j-1)}(2)=0 .
$$

By induction we have for $j=0, \ldots, r-1, k=1,2$,

$$
\begin{aligned}
F_{i}^{(j)}(k) & =0, & i & =1, \ldots,\lceil r / 2\rceil, \\
G_{i}^{(j)}(k) & =0, & i & =1, \ldots,\lfloor r / 2\rfloor .
\end{aligned}
$$

It remains only to show that the functions $F_{i}, i=1, \ldots,\left\lceil\frac{r}{2}\right\rceil, G_{i}, i=$ $1, \ldots,\left\lfloor\frac{r}{2}\right\rfloor$, are linearly independent. For $i=1, \ldots,\left\lceil\frac{3 r}{2}\right\rceil-j, F_{i, j}(x)$ is a linear combination of

$$
N_{i-1}^{2 n+1}(2 x), \ldots, N_{i+j-1}^{2 n+1}(2 x), N_{3 r-i-j}^{2 n+1}(2 x), \ldots, N_{3 r-i}^{2 n+1}(2 x) .
$$

We shall show by induction on $j$ that the coefficient of $N_{i-1}^{2 n+1}(2 x)$ for $F_{i, j}(x)$ is nonzero for $i=1, \ldots,\left\lceil\frac{3 r}{2}\right\rceil-j$. This is certainly true for $j=0$. Suppose that it is true for some $j, 0 \leq j \leq r-1$. In the derivation of (5.9) and (5.10) we have shown that

$$
F_{i, j}^{(l)}(k)=0, \quad i=1, \ldots,\lceil 3 r / 2\rceil-j, l=0, \ldots, j-1, k=1,2 .
$$

So by the Schoenberg-Whitney Theorem we cannot have $F_{i, j}^{(j)}(k)=0, k=1,2$, and so by symmetry,

$$
F_{i, j}^{(j)}(1) \neq 0 \neq F_{i, j}^{(j)}(2), \quad i=1, \ldots,\lceil 3 r / 2\rceil-j .
$$

From (5.7) and (5.8) and our inductive hypothesis, the coefficient of $N_{i-1}^{2 n+1}(2 x)$ for $F_{i, j+1}(x)$ is nonzero, and our inductive proof is complete.

Now suppose $\sum_{i=1}^{[r / 2]} a_{i} F_{i}=0$. Expanding as a linear combination of $N_{0}^{2 n+1}(2 x), \ldots, N_{3 r-1}^{2 n+1}(2 x)$, we see that $F_{1}$ is the only term involving a nonzero multiple of $N_{0}^{2 n+1}(2 x)$, and hence $a_{1}=0$. Continuing in this way gives $a_{i}=0$, $i=1, \ldots,\left\lceil\frac{r}{2}\right\rceil$. Thus $F_{i}, i=1, \ldots,\left\lceil\frac{r}{2}\right\rceil$, are linearly independent.

Similarly $G_{i}, i=1, \ldots,\left\lfloor\frac{r}{2}\right\rfloor$, are linear independent. But since the functions $F_{i}$ are even about $\frac{3}{2}$ and the functions $G_{i}$ are odd about $\frac{3}{2}$, we must have $F_{i}, i=1, \ldots,\left\lceil\frac{r}{2}\right\rceil$, and $G_{i}, i=1, \ldots,\left\lfloor\frac{r}{2}\right\rfloor$ are all linearly independent.

We now write

$$
\begin{array}{ll}
f_{i}=F_{i}^{(2 r)}, & i=1, \ldots,\lceil r / 2\rceil, \\
g_{i}=G_{i}^{(2 r)}, & i=1, \ldots,\lfloor r / 2\rceil .
\end{array}
$$

Then the functions $f_{i}$ and $g_{i}$ are respectively even and odd about $\frac{3}{2}$. From Lemma 5.1 we immediately have 
Theorem 5.1. The functions $f_{i}, i=1, \ldots,\left\lceil\frac{r}{2}\right\rceil, g_{i}, i=1, \ldots,\left\lfloor\frac{r}{2}\right\rfloor$, form $a$ basis for $\operatorname{span}\left\{\psi_{0}, \ldots, \psi_{r-1}\right\}$.

Remark 2. By Theorem 5.1 we can replace the functions $\psi_{j}, j \in \mathbf{Z}$, in the decomposition (4.1) by the functions $f_{i}(x-\nu), i=1, \ldots,\left\lfloor\frac{r}{2}\right\rceil, g_{i}(x-\nu)$, $i=1, \ldots,\left\lfloor\frac{r}{2}\right\rfloor, \nu \in \mathbf{Z}$.

\section{REFERENCES}

1. C. deBoor, Splines as linear combination of B-splines, Approximation Theory II (G. Lorentz, C. Chui and L. Schumaker, eds.), Academic Press, New York, 1976, pp. 1-47.

2. $\ldots$, Total positivity of the spline collocation matrix, Indiana Univ. J. Math. 25 (1976), 541-551.

3. Charles $\mathrm{K}$. Chui and Jian-ziong Wang, $A$ cardinal spline approach to wavelets, CAT Report \#211, Texas A\&M Univ., College Station, 1990.

4. __, A general framework of compactly supported splines and wavelets, CAT Report \#219, Texas A\&M Univ., College Station, 1990.

5. L. Daubechies, Orthonormal bases of compactly supported wavelet, Comm. Pure Appl. Math. 41 (1988), 909-996.

6. T. N. T. Goodman, S. L. Lee, and W. S. Tang, Wavelets in wandering subspaces, Trans. Amer. Math. Soc. (to appear).

7. P. R. Halmos, A Hilbert space problem book, 2nd ed., Springer-Verlag, 1982.

8. P. G. Lemaire, Ondelettes a localisation exponentielles, J. Math. Pures Appl. 67 (1988), 227-236.

9. S. Mallat, Multiresolution approximations and wavelet orthonormal bases of $L^{2}(\mathbf{R})$, Trans. Amer. Math. Soc. 315 (1989), 69-87.

10. J. B. Robertson, On wandering subspaces for unitary operators, Proc. Amer. Math. Soc. 16 (1965), 233-236.

11. I. J. Schoenberg, Cardinal spline interpolation, CBMS-NSF Series in Appl. Math., no. 12, SIAM Publ., Philadelphia, PA, 1973.

12. I. J. Schoenberg and A. Whitney, On Pólya frequency function. III. The positivity of translation determinants with application to interpolation problems by spline curves, Trans. Amer. Math. Soc. 74 (1953), 246-259.

Department of Mathematics and Computer Science, University of Dundee, Dundee DD1 4HN, SCOTLAND, UK

E-mail address: tgoodman Omcs.dundee.ac.uk

Department of Mathematics, National University of Singapore, 10 Kent Ridge CresCENT, SINGAPORE 0511

E-mail address: matleesl@leonis.nus.sg 